\title{
The Quantum Hydrodynamic Smooth Effective Potential
}

\author{
CARL L. GARDNER ${ }^{* \dot{亠}}$ and CHRISTIAN RINGHOFER ${ }^{\dot{*}}$ \\ Department of Mathematics, Arizona State University, Tempe, AZ 85287-1804
}

\begin{abstract}
An extension of the quantum hydrodynamic (QHD) model is discussed which is valid for classical potentials with discontinuities. The effective stress tensor for the QHD equations cancels the leading singularity in the classical potential at a barrier and leaves a residual smooth effective potential with a lower potential height in the barrier region. The smoothing makes the barrier partially transparent to the particle flow and provides the mechanism for particle tunneling in the QHD model.
\end{abstract}

Keywords: quantum hydrodynamic model, smooth effective potential

In his lectures on Statistical Mechanics, Feynman derives an effective quantum potential by a Gaussian smoothing of the classical potential. After demonstrating that the effective free energy based on the effective potential is accurate for smooth classical potentials like the anharmonic oscillator, he goes on to say that "it fails in its present form when the [classical] potential has a very large derivative as in the case of hard-sphere interatomic potential" - or for potential barriers in quantum semiconductor devices. In this note, we discuss an extension of Feynman's ideas to a smooth effective potential for the quantum hydrodynamic (QHD) model that is valid for the technologically important case of potentials with discontinuities.

The QHD equations have the same form as the classical hydrodynamic equations:

$$
\frac{\partial n}{\partial t}+\frac{\partial}{\partial x_{i}}\left(n u_{i}\right)=0
$$

$$
\begin{aligned}
& \frac{\partial}{\partial t}\left(m n u_{j}\right)+\frac{\partial}{\partial x_{i}}\left(m n u_{i} u_{j}-P_{i j}\right)=-n \frac{\partial V}{\partial x_{j}}-\frac{m n u_{j}}{\tau_{p}} \\
& \frac{\partial W}{\partial t}+\frac{\partial}{\partial x_{i}}\left(u_{i} W-u_{j} P_{i j}+q_{i}\right)=-n u_{i} \frac{\partial V}{\partial x_{i}}-\frac{\left(W-\frac{3}{2} n T_{0}\right)}{\tau_{w}} .
\end{aligned}
$$

where repeated indices are summed over and where $n$ is the particle density, $\mathbf{u}$ is the velocity, $m$ is the particle mass, $P_{i j}$ is the stress tensor, $V$ is the classical potential energy, $W$ is the energy density, $\mathbf{q}$ is the heat flux, and $T_{0}$ is the ambient temperature. Collision effects are modeled by the relaxation time approximation, with momentum and energy relaxation times $\tau_{p}$ and $\tau_{w}$. Quantum effects enter through the expression for the stress tensor (and for the energy density derived from the stress tensor).

Originally the quantum correction to the stress tensor in the QHD equations was given to $\mathrm{O}\left(\hbar^{2}\right)$ and involved second derivatives of the classical potential. In the spirit of Feynman, Ferry and Zhou [1] derived a

* Research supported in part by the U.S. Army Research Office under grant DAAH04-95-1-0122.

† Corresponding author. E-mail gardner@math.la.asu.edu

$\leftarrow$ Research supported in part by ARPA under grant F49620-93-1-0062. 
smoothed quantum potential for the QHD equations by linearizing an equation for the equilibrium density matrix. The Feynman effective partition function involves a smoothed potential of the form

$$
V_{a^{2}}(x)=\int \frac{d y}{\sqrt{2 \pi a^{2}}} \exp \left\{-\frac{(x-y)^{2}}{2 a^{2}}\right\} V(y)
$$

where $\mathrm{a}^{2} \propto \beta \hbar^{2} / m$ and $\beta=1 / T$ is the inverse temperature. The Ferry-Zhou effective stress tensor involves the difference between the smoothed and the local quantum potential $-\hbar^{2} \nabla^{2} n / 8 m n+V$. Their smoothing function is of the form $\exp \left\{-(\mathbf{x}-\mathbf{y})^{2} / 2 a^{2}\right\} /|\mathbf{x}-\mathbf{y}|$. Neither of these two approaches - which involve just a spatial averaging - has enough smoothing to handle discontinuities in $V$, since second spatial derivatives of the smoothed potential appear in both Feynman's free energy and the QHD equations.

To derive the new effective stress tensor and energy density, we construct an effective density matrix as an $O(\beta V)$ solution to the Bloch equation. Then using the effective density matrix, we take moments of the quantum Liouville equation to derive the QHD equations with the effective stress tensor and energy density [2].

The effective density matrix has the form

$$
\begin{aligned}
\rho(\beta, \mathbf{x}, \mathbf{y}) \approx & \left(\frac{m}{2 \pi \beta \hbar^{2}}\right)^{3 / 2} \\
& \exp \left\{-\frac{m}{2 \beta \hbar^{2}}(\mathbf{x}-\mathbf{y})^{2}-\beta \widetilde{V}(\beta, \mathbf{x}, \mathbf{y})\right\}
\end{aligned}
$$

where $\tilde{V}$ is given in center-of-mass coordinates

$$
\begin{aligned}
& \mathbf{R}=\frac{1}{2}(\mathbf{x}+\mathbf{y}), \mathbf{s}=\mathbf{x}-\mathbf{y} \text { by } \\
& \widetilde{V}(\boldsymbol{\beta}, \mathbf{R}, \mathbf{s})= \\
& \quad \frac{1}{2 \beta} \int_{0}^{\beta} d \beta^{\prime} \int d^{3} X^{\prime}\left(\frac{2 m \beta}{\pi\left(\beta-\beta^{\prime}\right)\left(\beta+\beta^{\prime}\right) \hbar^{2}}\right)^{3 / 2} \times \\
& \exp \left\{-\frac{2 m \beta}{\left(\beta-\beta^{\prime}\right)\left(\beta+\beta^{\prime}\right) \hbar^{2}} X^{\prime 2}\right\} \\
& \quad\left[V\left(\mathbf{X}^{\prime}+\mathbf{R}+\frac{\beta^{\prime}}{2 \beta} \mathbf{s}\right)+V\left(\mathbf{X}^{\prime}+\mathbf{R}-\frac{\beta^{\prime}}{2 \beta} \mathbf{s}\right)\right] .
\end{aligned}
$$

In constructing the effective density matrix, it is the change $\beta \delta V \equiv \beta(\max \{\mathrm{V}\}-\min \{V\})$ over a characteristic length scale that is assumed small rather than $\beta V$.

The change $\beta \delta V$ over a characteristic length scale may not be small at a potential step. In fact, for a 0.2 $\mathrm{eV}$ barrier, $\beta \delta V \approx 8$ at $300 \mathrm{~K}$ and $\approx 32$ at $77 \mathrm{~K}$. However, numerical comparisons demonstrate excellent agreement between the first three moments of the equilibrium full density matrix and the effective density matrix for the Bloch equation with a barrier potential for $\beta \delta V \lesssim 4$ (see Fig. 1), and good qualitative agreement for $\beta \delta V \lesssim 20$ (see Fig. 2). The moments of the $\mathrm{O}\left(\hbar^{2}\right)$ density matrix are in severe quantitative and qualitative disagreement with the moments of the full density matrix.

Using the effective density matrix in the moment expansion of the quantum Liouville equation, we

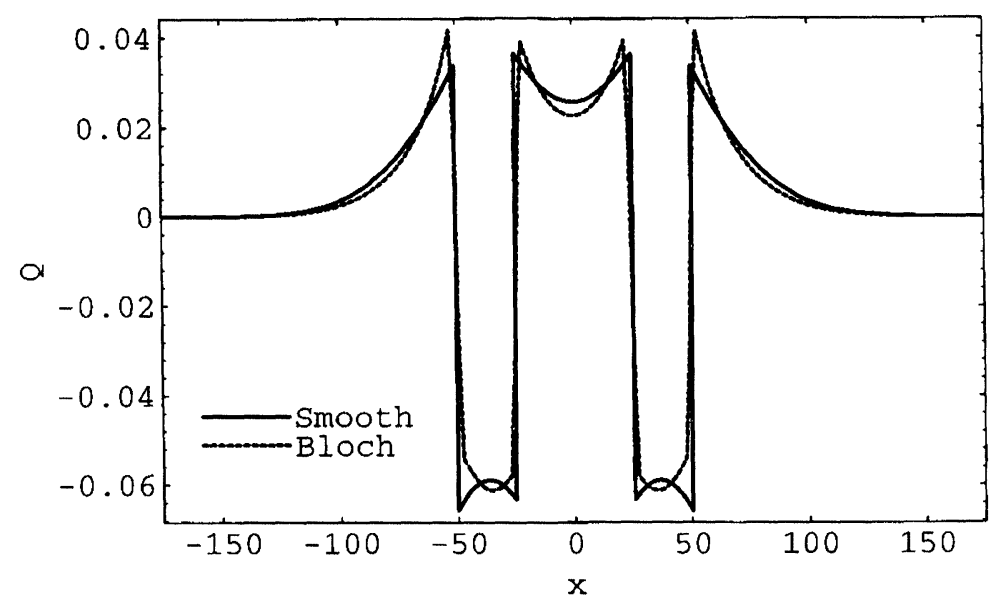

FIGURE 1 Quantum term in the energy in $\mathrm{eV}$ for electrons in a $0.1 \mathrm{eV}$ double barrier in GaAs at $T=300 \mathrm{~K}$. The barriers are $25 \AA$ and the well is $50 \AA$ wide. $x$ is in $\AA$ for all figures 


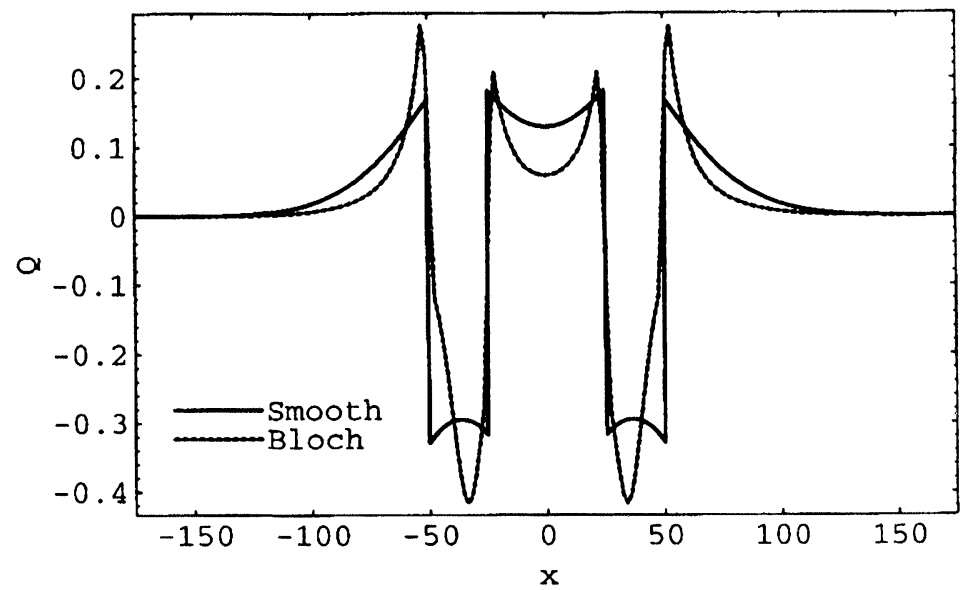

FIGURE 2 Quantum term in the energy in $\mathrm{eV}$ for electrons in a $0.5 \mathrm{eV}$ double barrier

obtain the QHD conservation laws as the first three moments with

$$
\begin{gathered}
P_{i j}=-n T \delta_{i j}-\frac{\hbar^{2} n}{4 m T} \frac{\partial^{2} \bar{V}}{\partial x_{i} \partial x_{j}} \\
W=\frac{3}{2} n T+\frac{1}{2} m n u^{2}+\frac{\hbar^{2} n}{8 m T} \nabla^{2} \bar{V}
\end{gathered}
$$

where the "quantum potential" is

$$
\begin{aligned}
& \bar{V}(\boldsymbol{\beta}, \mathbf{x})= \\
& \bar{\beta} \int_{0}^{\beta} d \beta^{\prime}\left(\frac{\beta^{\prime}}{\beta}\right)^{2} \int d^{3} X^{\prime}\left(\frac{2 m \beta}{\pi\left(\beta-\beta^{\prime}\right)\left(\beta+\beta^{\prime}\right) h^{2}}\right)^{3 / 2} \times \\
& \quad \exp \left\{-\frac{2 m \beta}{\left(\beta-\beta^{\prime}\right)\left(\beta+\beta^{\prime}\right) h^{2}}\left(\mathbf{X}^{\prime}-\mathbf{x}\right)^{2}\right\} V\left(\mathbf{X}^{\prime}\right) .
\end{aligned}
$$

The quantum correction to the classical stress tensor and energy density is valid to all orders of $\hbar^{2}$ and to first order in $\beta \delta V$, and involves both a smoothing integration of the classical potential over space and an averaging integration over temperature.

We define the 1D smooth effective potential in the momentum conservation equation (2) as the most singular part of $V-P_{11}$ :

$$
U \approx V+\frac{\hbar^{2}}{4 m T} \frac{d^{2} \bar{V}}{d x^{2}} .
$$

The double integration (over both space and temperature) provides sufficient smoothing so that the $P_{11}$ term in the smooth effective potential actually cancels the leading singularity in the classical potential at a barrier (see Fig. 3), leaving a residual smooth effective potential with a lower potential height in the barrier region. This cancellation and smoothing makes the barriers partially transparent to the particle flow and provides the mechanism for particle tunneling in the QHD model. Note that the effective barrier height approaches zero as $T \rightarrow 0$. This effect explains in fluid dynamical terms why particle tunneling is enhanced at low temperatures. As $T \rightarrow \infty$, the effective potential approaches the classical double barrier potential and quantum effects in the QHD model are suppressed.

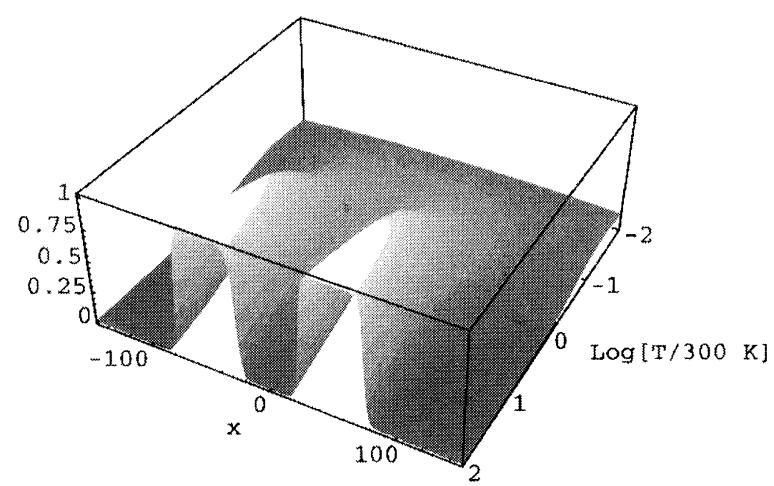

FIGURE 3 Smooth effective potential for electrons in GaAs for $50 \AA$ wide unit potential double barriers and $50 \AA$ wide well as a function of $x$ and $\log _{10}(T / 300 \mathrm{~K})$ 


\section{References}

[1] D. K. Ferry and J.-R. Zhou, "Form of the quantum potential for use in hydrodynamic equations for semiconductor device modeling," Physical Review, vol. B 48, pp. 7944-7950, 1993.

[2] C. L. Gardner and C. Ringhofer, "The smooth quantum potential for the hydrodynamic model," Physical Review, vol. E 53, pp. 157-167, 1996.

\section{Biographies}

Carl L. Gardner is Professor of Mathematics at Arizona State University. His current research interests lie in classical and quantum semiconductor device simulation and computational fluid dynamics.

Christian Ringhofer is Professor of Mathematics at Arizona State University. His current research interests include classical and quantum transport equations and moment models for semiconductor device modeling. 

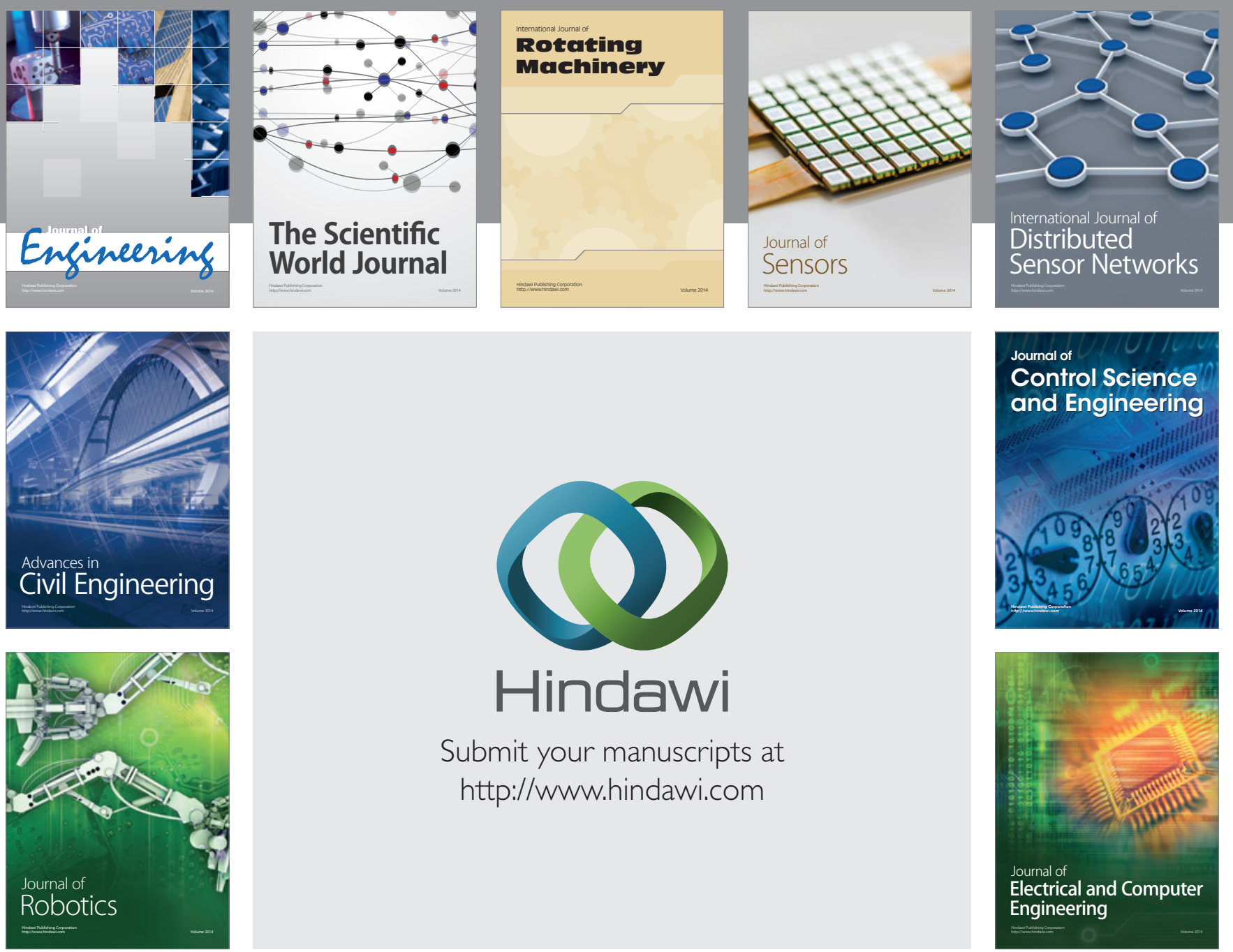

Submit your manuscripts at

http://www.hindawi.com
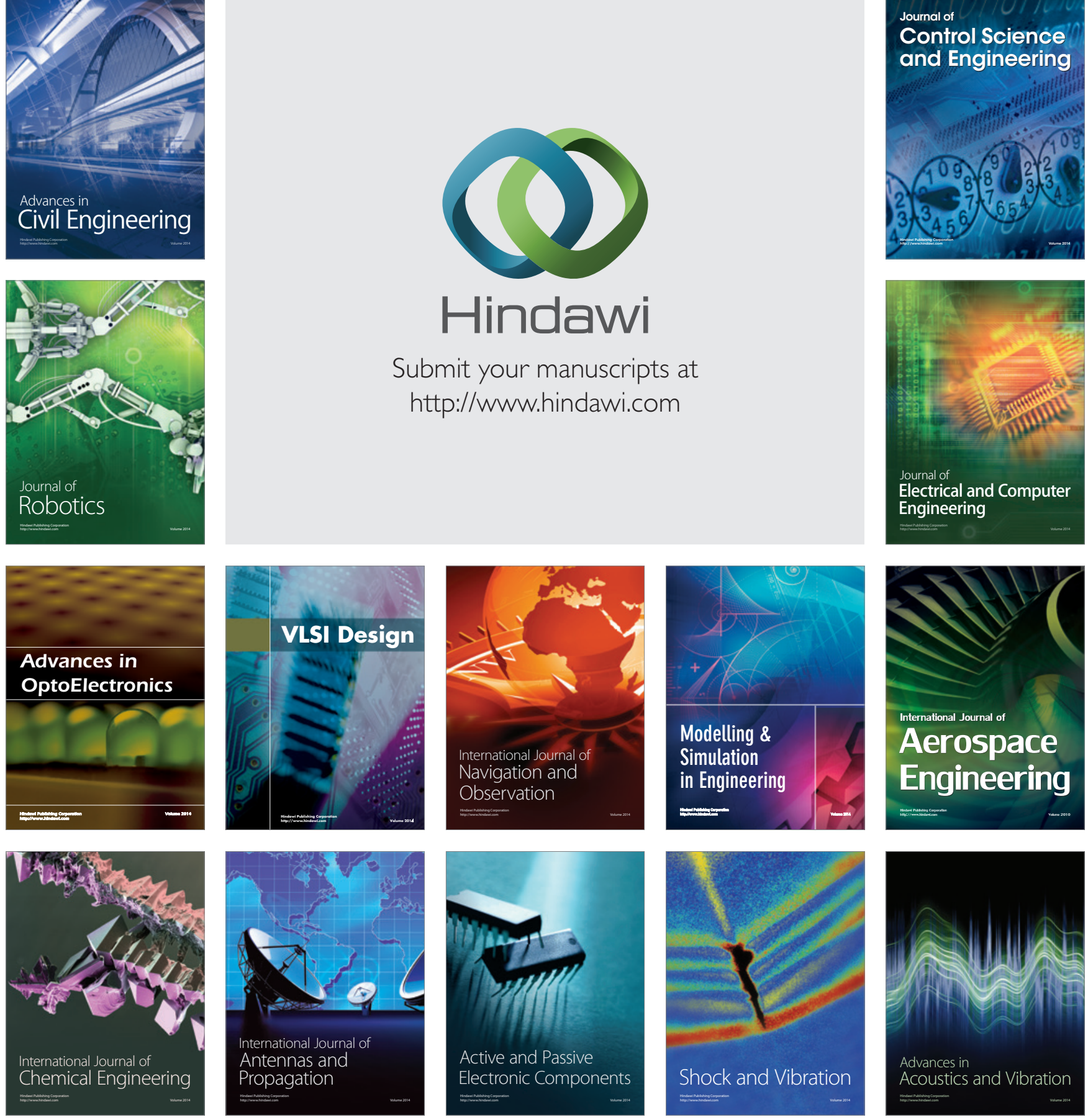